\title{
Evaluation of Biofilm and Enterotoxin Producing Capacity of Methicillin-resistant Staphylococcus aureus isolated from Healthy Persons
}

\author{
ANIE, CO \\ Department of Pharmaceutical Microbiology, Faculty Pharmacy Delta State University Abraka, Nigeria. \\ Corresponding author Email: oliseloke@yahoo.com Tel: 08066390868
}

\begin{abstract}
This study is aimed at investigating biofilm and enterotoxin producing capacity of methicillin-resistant Staphylococcus aureus isolated from nostrils of healthy students of Delta State University Oleh campus. Three hundred (300) nasal samples were collected using sterile swab stick and the isolates were identified using relevant biochemica tests. Determination of methicillin-resistant Staphylococcus aureus was carried out with the aid of Oxacillin sensitivity disc to test the presence of penicillin binding protein2a (PBP2a). The Methicillin-resistant Staphylococcus aureus (MRSA) isolates were screened to determine their biofilm producing ability using crystal binding assay. Latex agglutination test was carried out to detect the presence of enterotoxins among the MRSA isolates. The incidence of MRSA colonization among healthy individuals in the community of the $S$. aureus isolated was 47 (48.9\%). Enterotoxins B was mostly detected, only three Enterotoxin C were detected. All the MRSA isolates tested for the presence of biofilm showed positive results. It has been observed that MRSAs are capable of producing biofilms and enterotoxins which pose the risk antibiotic resistance and human Staphylococci toxicity.
\end{abstract}

\section{DOI: https://dx.doi.org/10.4314/jasem.v22i11.2}

Copyright: Copyright (C 2018 Anie. This is an open access article distributed under the Creative Commons Attribution License (CCL), which permits unrestricted use, distribution, and reproduction in any medium, provided the original work is properly cited.

Dates: Received: 22 October 2018; Revised: 20 November 2018; Accepted 27 November 2018

Keywords: Enterotoxins, Biofilm, Staphylococcus aureus.

Methicillin resistant staphylococcus aureus (MRSA) is known to be associated with several severe infections. The capacity of biofilm-formation decreases their susceptibility to many antibiotics. S. aureus is well known to form biofilms on diverse surfaces (Ando $e t$ al., 2004). Biofilms have an enormous impact on healthcare, and are estimated to be associated with $65 \%$ of nosocomial infections (Potera, 1999). Biofilms are the population of bacteria growing on the biotic and a biotic surface and embedded themselves in a self-produced extracellular matrix of exopolysaccharide (EPS), proteins and some micro molecules such as DNA. (Gowrishanker etal., 2012). Genus of Staphylococcus are known to be the commonest pathogens found in hospital surroundings, and they are the causative agents of a broad range of infections. Hospital-acquired infections have being the major source of morbidity and mortality as they are frequently acute and pyrogenic, and if untreated, they can cause bacteremia relating to a range of organs. In the genus of staphylococcus, S. aureus has always being the most significant specie in relation to hospital-a0cquired infections and continues to be the primary cause of community-acquired staphylococcus infection in many countries (Tenover and Gaynes, 2000). Outcome of infection clinically usually depend on the virulence nature of the pathogen concerned and the opposing efficacy of the defense mechanisms of the host. S. aureus usually express several potential factors of virulence. (Note: coagulase is generally not considered a virulence factor because coagulase/negative mutants are as virulent as the corresponding parent strains .The association between coagulase positivity and virulence in nature probably fortuitous).Many bacterial pathogens and nosocomial infection are the cause of Acute and chronic infections due to their ability to form biofilms (Stoodley et. al., 2002)The bacteria proliferation and the slime formation results in a higher resistance to antibiotics because drugs are prevented from reaching the bacteria that are protected by biofilms (Heilmanmet et al.,1996). Methicillin-resistant Staphylococcus aureus (MRSA) is one of the most important bacterial pathogens causing hospital-acquired infections and in recent years has become a serious cause of community onset infections as well (David et al., 2010). This study was to evaluate biofilm and enterotoxin Producing Capacity of Methicillin-resistant Staphylococcus aureus Isolated from Healthy Persons Delta State University Oleh campus.

\section{MATERIALS AND METHOD}

Materials And Culture Media: The material used includes; universal bottles, autoclave, hot air oven (model: GP/50/CLAD/250/HYD), incubator (model: GP/50/CLAD/250/HYD), cut glass slides, forceps, 
UV -visible spectrophotometer (LABTECH-2805), measuring cylinder, micro pipette, lid, double beam balance, centrifuge (model: 80-2) and wire loop. Nutrient agar, methanol, crystal violet, water, and sterile distilled water, Mueller-Hin-ton Agar, nutrient broth, Mannitol Salt agar.

Ethical Consideration: Samples were collected after; information as regards the study was thoroughly explained to the participants after approval was gotten after from the Institution Head. I obtained Oral consent prior to participation in the study.

Sample Collection of Bacteria Strains: Three hundred nasal samples were collected from the nares of healthy students in Delta state university with a sterile swab stick, which were carefully and gently inserted into the inner area and rubbed over the anterior nares of both nostrils. Samples were collected from March 2016 to July 2016

Phenotypic detection of Methicillin-resistant Staphylococcus aureus(MRSA):Oxacillin sensitivity disc was used to determine theSusceptibility of $S$ aureus isolates on Muller Hinton agar. The 0.5 Mcfarland standard $S$ aureus isolates was prepared. The standardized suspensions were aseptically spot inoculated onto the surface of Muller Hinton agar plates. The plates were incubated for 24 hours at $30{ }^{\circ} \mathrm{C}$. The inhibition zones were observed and recorded. These were interpreted as susceptible, intermediate and resistant according to standard specifications (Baker et al.., 2001).

Detection of Biofilm: The crystal violet binding assay techniques was used for the detection of the biofilm.Nutrient broth and universal bottles are sterilized by autoclaving for 15 minutes at $121^{\circ} \mathrm{c}$. The glass slides cut to the size of $4 \mathrm{~cm}$ by $1.4 \mathrm{~cm}$ by $0.1 \mathrm{~cm}$ each were washed with detergents, rinsed with sterile distilled water, and air dried before placing in a hot air oven at $160^{\circ} \mathrm{c}$ for 1 hour. Nutrient broth $(25 \mathrm{ml})$ was measured and poured into different universal bottles. The cut glass slides were picked aseptically using forceps and four were dropped into each universal bottle containing nutrient broth. The universal bottles containing nutrient broth were inoculated with Staphylococcus aureus isolated from different students in Institution and labeled accordingly. One sterile universal bottle containing nutrient broth not inoculated with Staphylococcus aureus serves as control. The broths inoculated were then incubated at $37^{\circ} \mathrm{c}$ for 24 hours. After 24 hours incubation, the set of glass slides were aseptically removed from the broth culture for biofilm quantification. Set of each glass slides were washed three consecutive times using $5 \mathrm{ml}$ sterile distilled water, and then fixed with methanol (3ml) per slide. Individual glass slide was stained with crystal violet and allowed to stand for 20 minutes and later flushed with water with running tap. Each glass slide was air dried and re-solubilized with $2.5 \mathrm{ml}$ of $33 \%$ glacial acetic acid in test tubes. Re-solubilized liquid was then emptied into cuvette and then absorbance determined against optical density of blank reading without inoculation of $S$. aureus (control) at a wave length of $620 \mathrm{~nm}$ using double beam UV-visible spectrophotometer. The negative control absorbance was subtracted from the absorbance of the re-solubilized solution containing $S$. aureus to determine the actual absorbance value. This procedure was repeated for another 3 batches of organisms (Staphylococcus aureus), each batch consisting of 10 samples. Pawar et al., (2005)

Detection of Enterotoxin: In other to detect enterotoxin from the samples, the reverse passive late agglutination method was used. Nutrient broth and universal bottles were sterilized by autoclaving and allowed to cool. Nutrient broth $(5 \mathrm{ml})$ was poured into each universal bottle and inoculated with Staphylococcus aureus with the aid of a wire loop. The inoculated broths were incubated for 24 hours at $37^{\circ} \mathrm{c}$. The centrifuge tubes, microtitre plate, lid and pipette tips were disinfected using disinfectants. The overnight broth culture of Staphylococcus aureus was poured into different centrifuge tubes, placed in a sample compartment and centrifuge for 20 minutes at 900rpm. After centrifugation, the culture supernatants were poured into different test tubes. The micro-titre plates were arranged so that each row contains 8 wells and each sample needs the use of five rows. Using a micro pipette, $0.25 \mathrm{ml}$ of enterotoxin detection diluents was dispensed in each well of the five rows; $0.25 \mathrm{ml}$ of test sample (staphylococcus aureus) was added to the first well of each five rows. Using a micro pipette and starting at the first well of each row, $0.25 \mathrm{ml}$ was picked up and doubling dilution was performed along each of the five rows; it was stopped at the $7^{\text {th }}$ well to leave the last well i.e. the $8^{\text {th }}$ well containing diluents only (positive control). To each well in the first row, $0.25 \mathrm{ml}$ of latex sensitized with enterotoxin A was added. To each well in the second row, $0.25 \mathrm{ml}$ of latex sensitized with anti-enterotoxin B was added. To each in the third row, $0.25 \mathrm{ml}$ of latex sensitized with anti-enterotoxin $\mathrm{C}$ was added. To each well in the fourth row, $0.25 \mathrm{ml}$ of latex sensitized with anti-enterotoxin $\mathrm{D}$ was added and finally, to each well in the fifth row, $0.25 \mathrm{ml}$ of latex control was added. The microtitre plate was agitated by hand to mix the content of each well while taking care to ensure that no spillage occurs from the wells. The plates are covered with a lid to avoid evaporation and allowed to stand in a vibration free surface at room temperature for 24 hours, each well in each row were 
examined against a black background for agglutination.

\section{RESULTS AND DISCUSSION}

Studies revealed that out of three hundred nasal samples collected from the study location and screened a total of 164(55\%) of isolates were found to be $S$. aureus, based on morphology and biochemical tests as shown table 1. Former studies have shown that an estimated $20 \%$ of the human populations are long-term carriers of $S$. aureus which has become potent risk factor for $S$ aureus infections (Vandenesch et al., 2003)

Table2: shows the incidence of MRSA from amonghealthy individuals in the community of the $S$. aureus isolated was $47(48.9 \%)$. Out of the three hundred (300) samples collected from the healthy students, forty-seven (47) isolates were known to be methicillin resistant $S$. aureus, that is, isolates resistant to oxacillin. Rate of colonization is an important step in the series of events that to infections caused by S.aureus (Ako-Nai et al., 1991)

Table1. Isolation frequency of $S$ aureus from healthy community individuals

\begin{tabular}{|l|l|l|l|}
\hline SOURCE & $\begin{array}{l}\text { Number } \\
\text { sampled }\end{array}$ & $\begin{array}{l}\text { Number } \\
\text { positive }\end{array}$ & $\begin{array}{l}\text { Percentage } \\
\text { positive }\end{array}$ \\
\hline TOTAL & 300 & 164 & 55 \\
\hline
\end{tabular}

Table 2: Incidence of MRSA among the healthy students DELSU Oleh

\begin{tabular}{|l|l|l|}
\hline Source & MRSA number & Percentage \\
\hline Total & 47 & 48.9 \\
\hline
\end{tabular}

Table 3: Thickness of Biofilm of the MRSA from healthy students of Oleh study location

\begin{tabular}{|l|l|l|}
\hline Isolates & Sample location & Biofilm thickness $(\mathrm{mm})$ \\
\hline 1 & OLEH & 0.03 \\
\hline 2 & OLEH & 0.05 \\
\hline 3 & OLEH & 0.03 \\
\hline 4 & OLEH & 0.04 \\
\hline 5 & OLEH & 0.02 \\
\hline 6 & OLEH & 0.04 \\
\hline 7 & OLEH & 0.03 \\
\hline 8 & OLEH & 0.03 \\
\hline 9 & OLEH & 0.03 \\
\hline 10 & OLEH & 0.05 \\
\hline 11 & OLEH & 0.05 \\
\hline 12 & OLEH & 0.02 \\
\hline 13 & OLEH & 0.05 \\
\hline 14 & OLEH & 0.03 \\
\hline 15 & OLEH & 0.05 \\
\hline 16 & OLEH & 0.03 \\
\hline 17 & OLEH & 0.04 \\
\hline 18 & OLEH & 0.04 \\
\hline 19 & OLEH & 0.04 \\
\hline 20 & OLEH & 0.04 \\
\hline
\end{tabular}

This study showed that the twenty (20) MRSA representative subjected to this test produced biofilm at varying capacities as shown in Table 3. Biofilms have known to be good sources of pathogen that cause persistent infections. They are responsible for much nosocomial infections and are also associated with many medical conditions including indwelling medical device, dental plaques, upper respiratory tract infections and urogenital infections (Afrenish, etal., (2001) ). MRSA isolates tested for the presence of biofilm showed positive results which is in line with earlier reports (Costerton et al., 1999).). Initial bacterial monolayer which sticks to polymeric substances has to change to common biofilm which includes bacteria and extra cellular slime substance. The proliferation of bacteria and formation of slime or biofilm results in higher resistance to an antibiotic because antibiotis are usually prevented from getting into the bacteria which are protected by slime or biofilm (Heilmann, et al., 1996).In medicine, the emphasis on capability of Staphylococcus aureus to form biofilm cannot be overemphasized since this biofilm is capable of promoting resistance to antimicrobials by shielding the microorganisms from antibiotic effects. Biofilm-producing bacteria usually produce infections that are difficult to eliminate. They manifest resistance to antibiotics by several methods which include restricted penetration of antibiotics into biofilm and expression of resistant gene.

Out of the twenty MRSA isolates subjected to this test most of them were Enterotoxins B with only three (3) Enterotoxin C were found. The Staphylococcal enterotoxins (SE) are known to cause food poisoning and induce vomiting and diarrhea from one to two hours after ingestion, but little has been acknowledged how Staphylococcal enterotoxins (SE) would have caused symptoms of food poisoning. The proposed mechanism was that they triggered an effect directly on the intestinal epithelium and on the vagus nerve, causing stimulation of the emetic center and increasing intestinal motility. On this back ground, it is now pertinent to determine the ability of $S$. aureus to produce enterotoxins. Reverse Latex Agglutination method was used in this study to detect the presence of $S$. aureus enterotoxins in cultures of supernatant (Igarashi etal., 1998).

Conclusion: This study has established the prevalence of methicillin resistant Staphylococcus aureus (MRSA) healthy persons in this community. MRSA isolated possessed the capacity to produce biofilm. The capacity to produce biofilm is of varying degree, with the biofilm thickness varying from one isolate to another. It has also been observed that MRSAs could produce enterotoxins. Since these microorganisms Staphylococcus aureus could produce biofilms and enterotoxins which pose the risk of antibiotic 
resistance and human Staphylococci toxicity, so there is need to observe proper, appropriate use of drugs and adequate hygiene.

\section{REFERENCE}

Afrenish, H; Javaid, U; Fatima, K; Maria, O; Ali, K; Muhammad, I (2001). Evaluation of different detection methods of biofilm formatiom in the clinical isolates. Bra. J. Infect. Dis. 15 (4): 14138670

Ako-Nai, NK; Ounniyi, AD; Lamikanra, A; Torimiro, SE (1991). The Characterization of clinical isolates in Ile-Ife. Microbiol. 34:109-112.

Ando, E; Monden, K; Mitsuhata, R; Kariyama, R; Kumon, H (2004). Biofilm formation among methicillin-resistant Staphylococcus aureus isolates from patients with urinary tract infection. Acta Med. Okay. 58 (4): 207-214.

Baker, FJ; Silverton, RE; Pallister, CJ; (2001). Baker and Silverton's Introduction to Medical Laboratory technology. $7^{\text {th }}$ Ed.Nigerian Ed. Sam -Adex Printer, Ibadan. p323.

Costerton, JW; Stewart, PS; Greenberg, EP (1999). Bacteria biofilm a common cause of persistent infection. Sci. 284, 1318-1322

David, MZ; Daum, RS (2010). Community-associated methicillin-resistant Staphylococcus aureus: epidemiology and clinical consequences of an emerging epidemic. Clin. Microbiol. 23: 616-687.

Gowrishankar, S; Duncun, M; Karutha, N; Pandian, S (2012) Coral-associated bacteria as a promising antibiofilm agent against methicillin-resistant and susceptible Staphylococcus aureus biofilms. Evid. Based Comp. Alt. Med. 862374.

Heilmann, C; Gerke, C; Pedreau-Remington, F; Gotze, F (1996). "Characterization of Tn917 insertion mutant of Staphylococcus epidermidis affected in biofilm formation," infect. Immune. 64(1): 277-282

Igarashi, H; Fujikawa, H; Shingaki, M; Bergdoll, S (1998). Latex agglutination test for staphylococcal toxic shock syndrome toxin. J. Clin. Microbiol. 23(3):509-12

Pawar, D; Rossman, M; Chen, J (2005). Role of curli fimbriae in mediating the cell of enterohaemorrhagicEsherichia coli to attach to abiotic surfaces. J. Appl. Microbiol.99: 418-425

Potera, C (1999). Forging a Link between Biofilms and

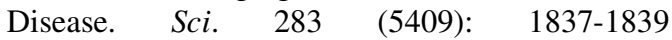

Stoodley, KP; Sauer, DG; Costerton, JW (2002). "Biofilm as complex differentiated communities," Ann. rev. microbiol. 56: 187-209

Tenover, FC; Gaynes, RP (2000). The epidemiology of staphylococcus infections. In: Fischetti VA (ed) Gram-positive pathogens. American Society for Microbiology, Washington DC, 414-419

Vandenesch, F; Naimi, T; Enright, MC (2003). Community-acquired methicillin-resistant Staphylococcus aureus carrying Panton-Valentine leukocidin genes: worldwide emergence. Emerge. Infect. Dis. 9: 978-84 